\section{Las Comunidades Terapéuticas como modelo alternativo para el proceso de inclusión social de pacientes con esquizofrenia}

Therapeutic Communities as an alternative model for the process of social inclusion of patients with schizophrenia

Sandra G. Rosas Landa Zamudio; Israel B. Aguillón Cruz

\section{RESUMEN}

Este artículo presenta y describe algunas de las líneas del trabajo que se realizan dentro de la Comunidad Terapéutica Casa de Medio Camino Querétaro. Se busca exponer sus alcances, limitaciones, así como las posibles vías de estabilización que se logran durante el proceso de inclusión social de pacientes con esquizofrenia. A través de este estudio de caso local en México, se plantean las dificultades, retos y construcciones que se viven al interior de una Comunidad Terapéutica como dispositivo social respetando la singularidad de cada caso. Se exponen y explican algunas de las dinámicas al interior de la Casa de Medio Camino Querétaro las cuales permiten efectos terapéuticos y productos concretos realizados por los usuarios de la comunidad en su proceso de inclusión social. También se exponen las variables que intervienen positiva o negativamente en el seguimiento del trabajo terapéutico y psicológico durante el periodo de tiempo que se encuentran como residentes. Lo anterior en definitiva no es sencillo e implica enormes esfuerzos de tipo cualitativo que son ejecutados por diferentes actores, así como el esfuerzo subjetivo del paciente de realizar un proceso personal para mantener su funcionamiento, autonomía y vínculos sociales.

Palabras clave: Casa de Medio Camino; Comunidad Terapéutica; Inclusión social; Esquizofrenia

\section{ABSTRACT}

This article presents and describes some of the lines of work carried out in the Therapeutic Community Casa de Medio Camino Querétaro. It seeks to expose its achievements, limitations, as well as ways of stabilization achieved during the process of social inclusion of patients with schizophrenia. Through this local case study in Mexico, the difficulties, challenges, and constructions that are experienced within a Therapeutic Community as a social device are presented, respecting the uniqueness of each case. Some of the dynamics within the Casa de Medio Camino Querétaro, which allow therapeutic effects and concrete products made by the users of the community in their process of social inclusion, are exposed and explained. It also exposes the variables that intervene positively or negatively in the follow-up of the therapeutic and psychological work during the period of time they are as residents. This is not simple and involves enormous qualitative efforts that are carried out by different actors, as well as the patient's subjective effort to carry out a personal process to maintain his or her functioning, autonomy, and social ties.

Keywords: Casa de Medio Camino; Therapeutic community; Social inclusion; Schizophrenia
RELIGACIÓN

REVISTA DE CIENCIAS SOCIALES Y HUMANIDADES JOURNAL OF SOCIAL SCIENCES AND HUMANITIES REVISTA DE CENCIAS SOCIAIS E HUMANAS

INFORMACIÓN:

http://doi.org/10.46652/rgn.v6i30.857 ISSN $2477-9083$

Vol. 6 No. 30, 2021. e210857 Quito, Ecuador

Enviado: octubre 02, 2021

Aceptado: noviembre 27, 2021

Publicado: diciembre 13, 2021

Publicación Continua

Sección Dossier | Peer Reviewed
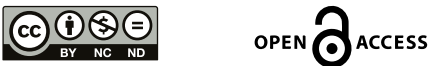

\section{AUTORES:}

(D) Sandra G. Rosas Landa Zamudio Universidad Nacional Autónoma de México - México sandrarolaz@gmail.com

(D) Israel B. Aguillón Cruz

Universidad Autónoma de Querétaro - México

israel.agc@gmail.com

\section{Conflicto de intereses}

Los autores declaran que no existe conflicto de interés posible.

Financiamiento

No existió asistencia financiera de partes externas al presente artículo.

\section{Agradecimiento}

Agradecemos a todos aquellos quienes han formado parte del equipo de colaboradores; huéspedes y familias que se han involucrado en el proceso de inclusión social de su familiar. Así como a la "Casa de Medio Camino QuerétaroLoohl".

Nota

Producto de la observación, seguimiento y trabajo cotidiano que se realiza dentro de la Comunidad Terapéutica Casa de Medio Camino Querétaro-Loohl, en diferentes periodos desde 2013 a la fecha.

\section{ENTIDAD EDITORA}




\section{Introducción}

El proceso de inclusión social para pacientes con esquizofrenia implica coordenadas clínicas y familiares, así como encrucijadas, imposibilidades y retos los cuales son indispensables identificar para lograr estabilizaciones de mayor tiempo, además de su apego y adherencia al tratamiento médico-psiquiátrico.

En el escenario contemporáneo aún existen barreras como el estigma (Stucchi, 2013) de la enfermedad mental, especialmente de pacientes con esquizofrenia. Las creencias culturales heredadas por modelos asilares y asistenciales han dejado una huella profunda para pensar el trato y formas de abordaje de estos pacientes. La consolidación histórica respecto al imaginario colectivo (De Certeau,1996) sobre los paradigmas de prácticas (De Certeau, 1995) que el tratamiento desde la psiquiatría propuso -entre ellos los asistenciales- siguen siendo piedra angular en las consideraciones para el tratamiento de la enfermedad mental, centrándose en debatir la compleja acción terapéutica y priorizando las discusiones sobre los diferentes modelos de tratamiento (Capponi, 2011) las cuales se enfocan en demostrar su efectividad. Sin embargo, la mayoría de las veces excluyen la singularidad, subjetividad y recursos con las que cuenta el paciente y su familia respecto a su situación particular, temporal, económica y socio-cultural que les brinde un panorama más amplio de soluciones-opciones distintas para una mejor calidad de vida. Al día de hoy las consecuencias heredadas por el estigma cultural sobre la enfermedad mental siguen siendo significativas principalmente vinculadas a conceptos como: encierro, institucionalización, asedio, maltrato, ataduras físicas, violaciones a los derechos humanos, locura, anormalidad.

La Castañeda, en la cultura mexicana es un referente social estrechamente ligado a la enfermedad mental, siendo el centro psiquiátrico más grande de México hasta la segunda mitad del siglo XX. Gracias a las recientes investigaciones de historiadores, sociólogos y filósofos quienes han puesto su foco de atención en rastrear las huellas (Derridá, 1972) dejadas por las prácticas y efectos de la psiquiatría hasta antes de los años setenta (Ríos, 2016), han permitido realizar análisis más críticos, pero sobre todo poner en relieve los modos en que dichas prácticas fueron cimientos en el tratamiento de las enfermedades mentales en el país. Así mismo es indispensable reconocer que al día de hoy ese ejercicio nos permite la reflexión y acción para re-pensar, replantear y re-orientar dichas prácticas.

En la actualidad las propuestas internacionales (Organización Mundial de la Salud, 2011) plantean modelos diferentes y alternativos al encierro. Entre ellos los de las Comunidades Terapéuticas. Su historia es reciente, datan de finales de los cincuenta. Maxwell Jones, en 1956 estableció la importancia y efectos positivos que tiene la Comunidad Terapéutica al potencializar y priorizar el trabajo grupal como soporte y apoyo entre los mismos pacientes, asimismo describió las dificultades y complejidades que el modelo implica para afrontar las resistencias inevitables frente a los cambios "La solución que ofrece la Comunidad Terapéutica es la libre discusión de la totalidad de las circunstancias por todos los comprometidos en ella, sin distinción alguna de títulos académicos, administrativos o jerárquico" (Jones, 1956). Ernest Simmel, desde 1920, ya hablaba y proponía este modelo para grupos de adictos. Su propuesta de trabajo terapéutico- 
clínico fue el psicoanálisis (Pérez del Río, 2009) en contraste, Jones, planteó como principio el trabajo grupal y ocupacional.

A finales de los sesenta en Europa surgió el movimiento antipsiquiátrico. Basaglia con su texto La institución negada. Informe de un hospital psiquiátrico (1972) hizo una crítica fundamental al sistema asilar y asistencialista que brindaban los hospitales psiquiátricos, cuestionando de fondo la mirada bio-médica que reduce al sujeto a categorías duales: de normalidad vs anormalidad, enfermedad vs salud, efectividad vs resistencia -al tratamiento-. Su propuesta dio un giro importante en la manera de concebir, cuestionar y analizar las intervenciones para los tratamientos para los pacientes con enfermedades mentales.

El Estado mexicano durante el periodo de 1960 a 1967, con la llegada de las nuevas reformas y transformaciones al interior de la antes llamada Secretaría de Salubridad y Asistencia -hoy su nombre Secretaría de Salud- se dividió en dos grandes modelos: "hospitales de alta especialidad para los casos agudos y subagudos y hospitales granja dirigidos a los enfermos crónicos bajo el ideario de comunidad terapéutica" (Sacristán, 2018, p. 149). Sin embargo, los esfuerzos principalmente de quienes estaban a cargo de los hospitales granja no se "valoraron en toda su dimensión cuando se desplegó el proyecto" (Tejeda Ruiz, 1967). El fracaso del modelo de las granjas se debió principalmente a la reproducción de experiencias de cronificación y confinamiento. A finales de la década de los setenta y principios de los ochenta la crítica periodística y académica cuestionó las condiciones en las que se encontraban los pacientes. La influencia de Basaglia, Laing y Cooper en México, fue significativa, un parteaguas para los jóvenes médicos y la academia.

Esta coyuntura se aprovechó para defender al hospital psiquiátrico como la institución moderna que contaba con las instalaciones adecuadas, personal adiestrado y programas de tratamiento y rehabilitación, por lo que no tenía ningún sentido promover su abolición, sino su transformación. Con el paso del tiempo y la firma por parte de México de la Declaración de Caracas (1990), inició un proceso hacia un mayor respeto de los derechos de los enfermos mentales. (Sacristán, 2018, p.151)

A cuatro años de la Declaración de Caracas se decretó la Norma Oficial Mexicana NOM-025SSA2-1994 para la prestación de servicios de atención integral hospitalaria médico-psiquiátrica. En 2003 el modelo propuesto por la Secretaria de Salud estableció como columna vertebral al tratamiento de pacientes psiquiátricos el modelo Hidalgo (Frenk, 2002). Éste propone la creación de nuevas estructuras de atención, en donde se pone énfasis en el respeto a los derechos de los usuarios de cualquier edad, la prevención, atención ambulatoria, hospitalización y reinserción social. Se considera un modelo comunitario de atención que promueve la gratuidad del servicio. El modelo Hidalgo, pone énfasis en el concepto de rehabilitación, reintegración y reinserción social a través de su red de servicios con distintas alternativas según las necesidades y especificaciones requeridas por el usuario. Coloca su acento en el concepto de modelo comunitario, como lo hicieron en su momento los modelos de hospitales granja. Sin embargo, la diferencia, radica principalmente en la ramificación y división de espacios para la atención, así mismo, el entorno y la comunidad en la que vive son parte esencial para su proceso de reintegración social. 
Se trataría de un espacio físico, arquitectónico y estructuralmente integrado entre sí, en donde cada una de las piezas que lo forman permiten interacción, multidisciplinaridad y acciones enfocadas a las necesidades de los pacientes y sus familias. Sin embargo, al día de hoy éste no se ha concretado a nivel nacional debido a la tendencia al tratamiento asilar, asistencial, médica y enfocado a la prevención.

Han sido amplios los esfuerzos tanto de médicos como de activistas y sociedad civil por migrar a modelos más eficientes en los servicios de atención a la salud mental. Sin embargo, el presupuesto y los costos para consolidar los servicios de atención son insuficientes. Según el Informe de Evaluación de Salud en México de la OMS revela que "el $2 \%$ del presupuesto total del recurso federal se destina para la Salud Mental, y de ese porcentaje el 80\% se destina para gastos de los hospitales psiquiátricos" (2011). Estos datos reflejan el mínimo de apoyo gubernamental que se destina a la atención de la salud mental. Ante ello, las instituciones públicas responden a las necesidades políticas, económicas y sociales, más que a las necesidades del paciente y su familia.

En 2013, el Programa de Acción Específico de Salud Mental propuso en sus lineamientos que "La hospitalización de estancias cortas además del proceso clínico farmacológico trabaje con programas de rehabilitación psicosocial, siempre pensando en la reinserción del usuario en su comunidad" (PAE, 2013). Sin embargo, son pocos los centros de atención que realizan estas prácticas o se enfocan principalmente en el trabajo ocupacional temporal del paciente. El modelo Hidalgo, a pesar de ser una propuesta para el tratamiento la atención de pacientes psiquiátricos integral, no se ha logrado consolidar. En algunos casos, los hospitales psiquiátricos terminan convirtiéndose en asilos o albergues donde se confinan a las personas con enfermedades mentales

Durante los esfuerzos que ha promovido México sobre los problemas de atención especifico en salud mental se encuentra erradicar las violaciones a los derechos humanos, así como las prácticas de tortura en los usuarios y usuarias que se encuentren hospitalizados en las unidades medico psiquiátricas considerando que tan solo en el 2013 el último reporte de la Comisión Nacional de Derechos Humanos, señalo que en los hospitales psiquiátricos públicos, se cometen faltas en detrimento de los derechos humanos de los usuarios por ello pensar la reclusión de las personas que han atravesado una experiencia psiquiátrica y apuntarla a una dirección donde se dignifique y dé una amplitud mayor calidad de vida del paciente y sus familiares ( PAE, 2013).

El principal conflicto es el seguimiento al tratamiento y a los programas de reinserción social; las estrategias más comunes son la promoción, prevención, talleres ocupacionales y los grupos de apoyo terapéutico, sin embargo, éstos suelen establecerse con grupos reducidos, así como en periodos breves de tiempo y en los cuales se guía al paciente a realizar actividades que no necesariamente lo acompañan de manera individual en sus procesos subjetivos, cognitivos, psicosociales, así como de intereses-habilidades personales. Están sujetos a responder a las políticas públicas en salud mental, generando una problemática compleja en su interior ya que no cuentan con los recursos humanos, económicos y materiales (IESM-OMS, 2011) necesarios, 
esto complejiza tanto la atención, seguimiento y apoyo integral para el paciente, su familia y el cuidador primario.

\subsection{Modelos y Dispositivos para el tratamiento de pacientes con esquizofrenia}

Cuando pensamos en enfermedad mental nos enfocamos en el paciente como el que padece y requiere de atención, empero, la familia y/o el cuidador primario la mayoría de las veces, también vive una situación desalentadora, estresante y muchas veces en solitario que genera y propicia situaciones de encierro involuntario- la vida cotidiana se ve fracturada por estar al cuidado de su familiar y las relaciones se desgastan a tal punto que llegan a ser violentas y no permite movilizarse a generar dinámicas diferentes- como respuesta a las creencias socio-culturales, las cuales conllevan también a complejas interacciones en la dinámica familiar gestando un circuito de repetición en patrones de conductas, hábitos y respuestas que se ciclan al interior del sistema familiar. Preguntas como ¿a dónde acudir?, ¿quién o cómo se le va a atender?, ¿qué diagnóstico se tiene?, ¿cuáles son los costos?, ¿cuáles son las causas?, ¿se va a curar? son preguntas frecuentes para las familias que buscan respuesta a la situación real que atraviesan. Si bien existen los espacios de atención a la salud mental, las instituciones públicas se enfocan principalmente en responder a la prevención, contención y rehabilitación parcial-momentánea para atender y dar solución al problema inmediato. Los espacios de contención física son una solución parcial frente al tema de salud mental y tiene que ver con la posibilidad y los recursos económicos con los que cuenta cada familia para sostener el tratamiento.

En México, existen distintos tipos de dispositivos privados que permiten al paciente abordar y coadyuvar a dar respuesta -temporal- a las necesidades específicas que tanto él como su familia requieren resolver. Cada dispositivo presenta una alternativa, la cual es necesaria para dar seguimiento al tratamiento del paciente, cada uno, desde su saber y enfoque plantea prácticas específicas. Los hospitales, clínicas, anexos, casas de medio camino -sobre ello en adelante se especificará su funcionamiento- y centros de día se han fundado con finalidades distintas, sin embargo, cada dispositivo responde a los familiares y pacientes psiquiátricos desde marcos funcionales, ideológicos, filosóficos y éticos por los que fueron fundadas.

Los hospitales psiquiátricos operan desde una infraestructura bio-médica para estabilizaciones farmacológicas y de corta estancia. Éstos son necesarios para lograr la regulación farmacológica, así como el seguimiento y meticuloso monitoreo médico especializado. En la Norma Oficial Mexicana NOM-025-SSA2-2014 se establecen los lineamientos para el internamiento del paciente, responde y considera los derechos del paciente y el actuar del médico frente al tratamiento. Ello ha permitido en gran medida centrar las bases para alcanzar la congruencia, así como dignificar el trato y derecho como persona del paciente.

Otros espacios que dan respuesta inmediata desde la contención física y proporcionan internamientos de pacientes con padecimientos psiquiátricos son los anexos. En México éstos establecen su práctica orientada principalmente en controlar la conducta a través del aislamiento, coerción y contención forzada, recrean modelos de encierro para el proceso de rehabilitación del sujeto frente al consumo y la abstinencia. Este modelo -en algunos casos- todavía centra 
su filosofía en bases religiosas, así como en el seguimiento de los 12 pasos de alcohólicos anónimos. Aunque su forma de trabajo no aplica para pacientes psiquiátricos, son dispositivos que garantizan a la familia el aislamiento y la contención frente "al descontrol" y temor cuando el paciente reacciona activo-agresivo debido a las alucinaciones o a la falta de adherencia al tratamiento farmacológico. Los anexos han funcionado como espacios de control y contención física, sin embargo, las familias, aunque identifiquen que, no son espacios diseñados para el paciente psiquiátrico acuden debido a la necesidad inmediata de evitar ya sea una situación de agresión directa, ya sea por su preocupación, impotencia y/o desesperación frente a la falta de apoyos. También destaca como factor predominante la situación económica de la familia para dar seguimiento al tratamiento, pues en la mayoría de los anexos las aportaciones económicas son más accesibles que en una clínica u hospital privado. Éstos han sido una solución para las familias ante la enfermedad mental. Los anexos son creados con finalidades específicas: trabajan con la abstinencia, dependencia y regulación en el consumo de sustancias.

Cada dispositivo tiene una función específica para los pacientes, aunque se han discutido, debatido y criticado las condiciones en que se tienen a los pacientes en hospitales, anexos, asilos, albergues y/o clínicas, cada uno de ellos brinda un soporte determinado a las circunstancias y necesidades requeridas del paciente y su familia. Por tanto, se reconoce y considera indispensable la relevancia y función que cada dispositivo proporciona; cada uno con sus limitaciones y alcances específicos.

La mayoría de los modelos terapéuticos, presentan una estructura la cual alude a prácticas de un espacio cerrado o potencialmente disciplinario, en donde se coartan las posibilidades del acceso a una mejor calidad de vida, una vida digna de ser vivida (Butler, 2010). Sin embargo, existen espacios en donde se promueve la singularidad subjetiva de los pacientes con enfermedades mentales.

Las casas de medio camino, son un espacio en donde se busca reintegrar al paciente a actividades ocupacionales y psicosociales. Un punto intermedio entre la hospitalización y la casa de origen del paciente. La influencia del ambiente y los factores sociales que forman parte de su entorno son piezas clave para que éste pueda encontrar vías de funcionamiento social. Los problemas de salud mental además del tratamiento médico requieren de otras esferas que conforman la vida cotidiana, afectiva, interpresonal y familiar. Las casas de medio camino son una alternativa que busca promover acciones que reduzcan factores de riesgo del ambiente emocional y psicosocial. El acompañamiento terapéutico y el soporte en la convivencia para que el paciente pueda retomar actividades y su funcionamiento permiten de manera moderada estabilizaciones de mayor tiempo. Este dispositivo tiene su centro de gravedad en la disposición arquitectónica y espacial. Es decir, se encuentran en el corazón de las ciudades, al interior de la actividad social. A diferencia de los hospitales y anexos, que, en su momento, fueron construidos en las afueras o periferias de las ciudades; las casas de medio camino se encuentran al interior de la dinámica social. Ello parte del principio de reintegrar y reincorporar al paciente a la experiencia social de manera paulatina. 
Quienes las integran son principalmente psicólogos clínicos, sociales, educativos y trabajadores sociales quienes buscan promover el desarrollo de habilidades e intereses del paciente. El esfuerzo que ello con lleva es enorme, ya que uno de sus principales problemas es caer en la cronificación del paciente y su asistencia. Su función es la de incorporar al paciente a la comunidad. Sin embargo, queda claro para quienes trabajamos en el epicentro de este modelo que ello no es posible sin el trabajo en conjunto, multidisciplinario y el involucramiento directo de las familias. La comunicación entre el psiquiatra, los psicólogos y la familia son necesarios para el logro de objetivos en común.

Las casas de medio camino, aunque originalmente forman parte de la propuesta del modelo Hidalgo, la mayoría de éstas en México son privadas. La razón radica principalmente en el presupuesto, recursos, tiempo y atención terapéutica que se les proporciona a los pacientes. El número de personas que residen en ella es reducido. El dispositivo plantea como línea de trabajo el ingreso y permanencia voluntaria del paciente.

Es indispensable señalar que una casa de medio camino como dispositivo, no necesariamente se orienta en trabajar desde un modelo de comunidad terapéutica; son pocas las instituciones en México que promueven y trabajan desde ese eje. La presencia, el cuerpo de un otro que está acompañando en el terreno resbaladizo que implica el trabajo con pacientes con esquizofrenia se logra con un trabajo minucioso y constante, el cual tiene que ser formulado desde un proceso singular y colectivo. La comunidad terapéutica como modelo implica la autonomía, pero también la interpelación sistémica del grupo que la conforma. No se trata únicamente de realizar actividades con la finalidad de entretener u ocuparse, sino que entre varios se puedan realizar proyectos en común que permitan tender puentes de solidaridad, empatía, colectividad y reconocimiento. En ella se implica necesariamente el concepto de inclusión social.

\subsection{Diferencias: rehabilitación, reinserción e inclusión psicosocial.}

En este punto, es necesario detenernos a cuestionar el concepto de inclusión social, y sus diferencias en contraste con conceptos como: rehabilitación, reinserción o reintegración social, ya que se puede asociar a la consideración de que con ello se busca "normalizar" al sujeto, es decir, que se adapte a las dinámicas, expectativas y lineamientos que la sociedad y cultura establecen. Que el sujeto se adhiera a las ideas imaginarias de ser productivo, así como de cumplir las expectativas sociales que se esperan, entonces que, más allá de ser una carga social logre su adaptación.

El concepto de rehabilitación se encuentra estrechamente ligado a la discapacidad. Sin embargo, "La necesidad de unificar criterios, en ocasiones ha dado como resultado la utilización de terminología que con el tiempo se ha considerado estigmatizadora o peyorativa para las personas con discapacidad" (Crespo, 2003, p. 20). De ahí que el enfoque de la rehabilitación centre sus esfuerzos principalmente en promover las capacidades del paciente en el mejor contexto social posible. Establecer objetivos específicos asociados al funcionamiento, modificar conductas de riesgo, asesorar e informar a los cuidadores primarios para que tengan una adecuada interacción con el enfermo. En el seno del concepto de rehabilitación existen históricamente cargas 
simbólicas y de estigma profundos que gravitan en torno al funcionamiento, las limitaciones y las restricciones de participación.

El horizonte del que viene el concepto de reinserción, sienta sus bases desde la criminología y la relación del sujeto con la ley. Su objetivo parte del supuesto de lograr la reintegración social al medio al que pertenece, a través de diferentes estructuras comunitarias, con ello se implica facilitarle los recursos socio-comunitarios del ambiente para evitar su aislamiento y por lo tanto promover su convivencia con el medio en el que vive. En este punto es que se plantea la participación de los pacientes en actividades laborales. El lugar del sujeto en el mundo tendría desde ahí que adaptarse a las situaciones, al medio y a sus circunstancias.

Ahora bien, la diferencia central entre estos conceptos y el de inclusión psicosocial, es que el sujeto fue excluido del marco social. La exclusión implica que se incurrió en una serie de actos y acciones que lo dejaron fuera. Los saberes que se adhieren al discurso científico legitiman las diferencias entre aquellos sujetos que están fuera y dentro del marco social. En paralelo el estatus de legalidad y legitimidad brindan las pautas y líneas que permiten la convivencia, por lo tanto, la dependencia a un sistema. La exclusión es entonces producto de la misma lógica del sistema: ésta sólo protege a aquellos que se ajustan a un modo determinado de vida y aquel "que no quiere o no puede acceder a ese modo de vida se encuentra marginado por el juego mismo de las instituciones" (Foucault, 1978). Por lo tanto, la inclusión social implicaría necesariamente una ética de la alteridad (Sutton, 2006, p. 2). Reconocer al otro en esa alteridad irreductible a la lógica que se establece como estandarizada o impuesta por el discurso hegemónico.

Desde ahí, la relación entonces con el otro semejante, es una relación de extrañeza y apertura con la diferencia. En ese sentido, la inclusión implica que no necesariamente hay un "deber" de reconciliarse con el otro ni con su naturaleza, sino desde la alteridad, permitiendo que el otro hable desde su diferencia. Permitir que el otro hable desde su subjetividad: reconocer la realidad del sujeto marginado, que ha quedado fuera del margen social, dejando de lado el velo de la estigmatización y el discurso de víctima de sus circunstancias. La inclusión implicaría entonces una forma de la emergencia de otros discursos y ponerlos en circulación. Asimismo, el sujeto se implica en esa alteridad de reconocimiento de sus limitaciones, no por el Otro sino por él mismo. Ello a su vez recorre el sendero de una ética propia que no es sin que afecte a los otros que le rodean.

Las casas de medio camino, son una vía que permiten la interrelación de estos tres conceptos, entonces ¿esto implica una forma de establecer pautas para que el sujeto se organice desde una identidad de normalidad? Posiblemente sí. Sin embargo, la diferencia consideramos radica en la manera en cómo se aborda comunitariamente, grupalmente y sistémicamente desde su interior. Abrirse a la extrañeza y aceptar la alteridad de un discurso distinto no es una posición sencilla. De ahí que el modelo de comunidad terapéutica permite dar un giro radical a la manera en cómo se trabaja en el interior.

Sin embargo, si bien esa es una tendencia, cabe mencionar la importancia de identificar que en el estudio de caso que se presenta el núcleo duro al que se apunta es a la manera en cómo un sujeto puede desde su singularidad y vía el soporte grupal lograr desarrollar pequeñas invenciones 
que le permiten a su forma, habilidades y recursos subjetivos encontrar alternativas para el restablecimiento de sus lazos sociales con otros, así como trabajar y regular -en la medida de sus posibilidades- los bajos niveles de tolerancia a la frustración. Se exponen algunas de las líneas de trabajo que permiten crear puentes y conexiones <<entre >> lo social, la cultura y la salud mental desde la singularidad.

El objetivo de este artículo es dar testimonio (Derridá, 2001) de quienes trabajamos, sostenemos e interactuamos al interior de la comunidad terapéutica Casa de Medio Camino Querétaro los alcances y limitaciones clínicas-terapéuticas que se presentan entre pacientes, psicólogos y familias para restablecer el lazo social, familiar y ocupacional que en muchas de las ocasiones se fractura debido a las circunstancias en las que vive el paciente. Sin embargo, no todas las casas de medio camino se constituyen como comunidad terapéutica porque ello implica dinámicas de la organización como un todo utilizando los recursos inherentes de los individuos para resolver conflictos que se presentan en la misma (Jones, 1956).

Se menciona e identifica cómo el trabajo desde lo cotidiano, multidisciplinario y en red permite tanto al cuidador primario como al paciente estructurar el funcionamiento, cuidado personal y de autonomía, así como de sus vínculos sociales, familiares y ocupacionales. Se expone la importancia del establecimiento de una red de apoyo -en este caso el que brinda como soporte la comunidad terapéutica- así como las limitaciones a las que se enfrenta el sistema familiar, social y terapéutico frente a la carencia de otros dispositivos y modelos que permitan dar continuidad al proceso de inclusión social.

\section{Metodología}

En este artículo se describieron y expusieron, a través del testimonio de quienes trabajamos en el interior de la Casa de Medio Camino Querétaro, las interacciones y participación del trabajo clínico-terapéutico que los psicólogos desarrollamos como parte de las prácticas y actividades cotidianas que se realizan. Se describieron las principales dificultades que se presentan al trabajar y convivir diariamente con pacientes con esquizofrenia en su proceso de inclusión social. Se describieron los retos, la complejidad y acciones que implican para nosotros mantener dinámicas comunitarias, de estabilidad e interacción con los pacientes sosteniendo los rasgos que constituyen el modelo de una comunidad terapéutica.

El trabajo que se desempeña al interior buscó en todo momento, brindar atención personalizada, centrada en el paciente y su familia, así como promover la autonomía y la inclusión social de los pacientes con esquizofrenia. Lo anterior resulta complejo y de gran esfuerzo por diferentes razones; primero porque se trabaja de manera sistémica, es decir, se involucra a la familia, al médico tratante y los vínculos externos; segundo porque el trabajo es grupal-comunitario, pero desde intervenciones dirigidas a cada caso; tercero se trabaja de manera singular; cuarto, porque la convivencia es integrativa y en un cotidiano.

Es un artículo que expuso las principales características de una casa de medio camino como comunidad terapéutica. Se propone como una invitación para reflexionar sobre la complejidad, 
energía, dinamismo, convocatoria que implica la interacción-acción requerida para trabajar en el plano de lo cotidiano con pacientes con esquizofrenia. Se destaca la relevancia y necesidad de acciones y prácticas a ejecutar para mantener el vínculo con otros; sostener el lazo social implica la posibilidad de contribuir desde un ángulo distinto, en donde el propósito se descentraliza del entramado exclusivamente biológico.

La población que hace uso de este dispositivo es de un grupo mixto de diez personas -la mayoría varones- el perfil de los huéspedes -así se les considera y nombra en la comunidad-oscila de entre 19 a 56 años. De los cuales el diagnóstico principal es de esquizofrenia paranoide. Todos ellos bajo tratamiento farmacológico indicado por su medico tratante. Y quienes residen de manera voluntaria por un tiempo específico en la comunidad.

Casa de Medio Camino Querétaro (2013), es un dispositivo terapéutico y social, porque apoya y brinda herramientas al paciente a dar continuidad a sus actividades cotidianas, de auto-cuidado y para su autonomía. Durante su estancia y convivencia con otros, los huéspedes trabajan con factores de convivencia común que suelen representar frustración, sin embargo, la interacción y organización cotidiana posibilita vías de reconocimiento del otro y actividades ocupacionales, así como una estabilidad ante eventos de crisis.

Las líneas de trabajo comunitarias que se siguieron para el proceso de inclusión social, centrándonos principalmente en: actividades cotidianas y sus efectos, actividades colectivas y sus resultados a nivel comunitario. Así mismo, se enuncian los factores de mayor complejidad que influyen en las dinámicas comunitarias e individuales, tal es el caso de: las relaciones familiares, sociales y ambientales que afectan a los pacientes con esquizofrenia en sus procesos de autonomía y lazo social.

\section{Casa de Medio Camino Querétaro-Casa Loohl un dispositivo para la inclusión social de} pacientes con esquizofrenia.

La Casa de Medio Camino Querétaro- Casa Loohl, es una comunidad terapéutica que representa para los pacientes psiquiátricos una alternativa posterior a la intervención hospitalaria, así como un espacio que funge como un puente y para poner en pausa dinámicas establecidas entre el paciente, la familia y el cuidador primario. Costumbres, hábitos e interacciones entre el paciente y el sistema familiar se vician y atoran en conductas repetidas y cíclicas. El modelo es a puertas abiertas y giratorias.

Es frecuente en un paciente con esquizofrenia que las crisis sean periódicas, ya sea porque los fenómenos sociales, ambientales o contingencias de la vida, para algunos casos son factores que los sobrepasan o los recursos subjetivos con los que cuentan no les permiten seguir funcionando de la misma manera, por lo tanto, requiere de una red de apoyo que le permita encontrar un equilibrio después de haber roto sus vínculos sociales, es decir, después de una crisis.

La comunidad terapéutica presenta como parte de su esquema, el ingreso voluntario y el derecho a la elección del paciente a residir por un tiempo específico en ella; un pacto voluntario. Esto 
traba implicaciones. Primero, gracias a ello, al querer estar en la comunidad, permite que sea posible un trabajo clínico-terapéutico con ellos, pues su estancia en la comunidad es temporal. Se concibe como un proceso donde tendrán estabilización y retoman actividades sociales que previo a ingresar a la comunidad tenían suspendidas por diversos motivos.

Es a puertas abiertas y cuya base de socialización no es la coerción, ni la disciplina, ni de corte a las relaciones sociales, familiares u ocupacionales como ocurre con los internamientos psiquiátricos, asilos o anexos. Se trata de una espacio qué tiene disímiles actividades internas y externas; se sostiene sobre el respeto y los acuerdos entre todos. Se trata de un dispositivo propiamente comunitario en lo cotidiano en donde a cada uno se le respetan sus habilidades, intereses y formas únicas de estar en el mundo; el factor que anuda la interacción es el vínculo y relación entre diferentes sujetos, que comparten un padecimiento. Las lógicas de intervención y dinámicas al interior, contienen variables radicalmente diferentes a la consulta del uno a uno. En cuanto al ámbito familiar a pesar de ser un lugar donde residen los pacientes psicóticos, también se distancia del mismo en tanto hay un esfuerzo declarado y constante por identificar y no repetir las dinámicas familiares -en no pocos casos- son ellas mismas las que desencadenan una crisis o evaden la adherencia al tratamiento. En la Comunidad Terapéutica, Casa de Medio Camino Querétaro, se interviene, buscando exista <<una >> - manera singular de "hacer" y de arreglárselas con lo que es único de cada quien- forma diferente para el devenir de un caso.

Las actividades cotidianas están marcadas por ciertas normas que se reflejan en un reglamento el cual es entregado a cada miembro que se integra a la comunidad. De esta manera hay un rango de horas para levantarse, otro para hacer el desayuno, la higiene de las zonas comunes y las personales, los talleres, la realización de la comida de la cena, así como de actividades menores cómo sacar la basura, organizar la alacena, mantener el refrigerador limpio horario de televisión, de salida a la tienda. Se trata de actividades cotidianas cuya ejecución la realizan los propios huéspedes con la perspectiva de que, en su tránsito por la Casa de Medio Camino, puedan crear rutinas para aumentar un grado de autonomía y facilitar posteriormente su incorporación a circuitos sociales, familiares o a una vida independiente.

La función del personal clínico de poder coordinar en armonía las actividades propuestas para el cotidiano no es labor sencilla, ni actitud fácil de encontrar o sostener, pues se necesita una cierta sensibilidad clínica para lograr hacer ejercer la norma sin que esto conlleve efectos de desencadenamiento y/o suspicacia o a generar ideas de enamoramiento producidas por la transferencia terapéutica que se produce en la convivencia cotidiana, así como también es necesaria la firmeza como semblante de ejercicio de una ley, que se regula, sin ser amenazante, pero que se hace existir por medio del dispositivo comunitario, con el objetivo de que los huéspedes no abusen del cuidado que se les otorga, para por medio de manipulaciones, chantajes sobre su "enfermedad", evadan sus responsabilidades básicas. Se trata de toda una labor que en ocasiones no es un problema sostenido, otras deben considerarse como un imposible en el momento, y siempre requiere de la figura del psicólogo, quien muy atento y con recursos creativos clínicos a la mano pueda sortear de una manera particular que la ley/límite general pueda entrar en cada uno de los huéspedes. En definitiva, no hay una manera de hacer - nos referimos a que no existen protocolos de intervención aplicados a todos de la misma 
manera. En ello radica la complejidad del trabajo en lo cotidiano con pacientes con experiencias psiquiátricas- porque no hay una situación general, ni un huésped tipo, es necesario un escucha orientada y fina para identificar la especificidad de la situación y el caso, para tomar la decisión de una intervención lo más adecuada posible a cada caso.

Con esto nos referimos a que las normas del dispositivo comunitario, permiten que cada huésped, según la propia dinámica de su caso, establezca acuerdos para que se le contemplen ciertas actividades por fuera del reglamento, como, por ejemplo; salidas a cursos, actividades laborales, horarios de computadora, salidas a caminar o hacer alguna actividad física, o bien acuerdos para realizar actividades creativas o de ocio fuera de la comunidad, como salida al centro de la ciudad, eventos. La elección y sus consecuencias -causa-efecto- como uno de los ejes rectores de la actividad comunitaria, sin embargo, ello, se hace a partir de un acompañamiento desde la singularidad de cada caso. La función terapéutica clínica de esta manera de proceder es doble; por un lado, detiene los eventos maniacos que puede presentar un huésped, da marco y forma a sus necesidades subjetivas buscando contener el desbordamiento, muchas veces desestructurado que se presenta en las psicosis, $y$, por otro lado, permite también que se puedan adaptar a sostener rutinas y, con valor mayor, que ellos mismos puedan poco a poco hacerse cargo de sus actos en lo que refiere a consecuencia.

\subsection{Lo cotidiano y el lazo social con personas con esquizofrenia: un reto de colaboraciones en común.}

En Casa de Medio Camino Querétaro, se trabaja desde la singularidad del caso, sin embargo, al ser una comunidad terapéutica se busca engarzar el quehacer singular con el exterior. El rasgo singular de cada caso, presenta una serie de tensiones con lo que clínicamente se trabaja, incluyendo las dificultades por las experiencias que implican estar y convivir con el otro semejante cumpliendo ciertas normas y límites.

Los procesos de construcción colaborativa, son a través de la creación y propuestas vinculadas con el arte, sin embargo, no todos los huéspedes se suman a ella. Principalmente porque no todos ellos, tienen interés, habilidad o simplemente un medio educativo, pedagógico y/o familiar por el que éste haya sido trasmitido. Nos deja claro que el arte y/o la literatura no son necesariamente la vía de construcción estabilizadora para la psicosis, en todos los casos, sin embargo, es un recurso. Al ser un trabajo colaborativo, cada uno participa desde sus intereses y contribuciones, desde lo singular se respetan las invenciones, recursos subjetivos y habilidades de cada uno en los proyectos comunitarios.

Se realizan tanto proyectos comunitarios como proyectos individuales. En el caso de los primeros permiten restablecer vínculos sociales y familiares, así como el reconocimiento social en dos vías: la de un otro "externo" que funge como testigo de la acción colectiva y la de los mismos compañeros, familia y red de trabajo de los profesionales quienes se encuentran en la Comunidad Terapéutica. 
El trabajo colectivo es posible únicamente gracias al apoyo, coordinación, disposición y creatividad del equipo de psicólogos, su participación es guía y puente para la ejecución de los proyectos. El involucramiento de los psicólogos dentro de la comunidad en lo cotidiano es pieza fundamental para la realización y culminación de cada proyecto. Ello en ocasiones resulta tarea compleja pues requiere de comunicación, tolerancia, respeto, interacción y cohesión como equipo de trabajo, así como el compromiso de cada uno de los miembros que lo conforman, el trabajo con pacientes psiquiátricos requiere de constancia, compromiso, transferencia y de una práctica cotidiana: actitudes y competencias que, no son comunes en la formación académica de los psicólogos. Son rasgos que se van forjando a partir de la convocatoria, compromiso y trabajo en conjunto.

Los talleres que se realizan dentro de la comunidad permiten también la gestación de proyectos en colectivo. El rol que adquieren los talleristas es relevante para los huéspedes como parte del equipo de trabajo, aunque son externos. De ellos se desprenden proyectos comunitarios, así como el establecimiento de redes de apoyo para los huéspedes. Cada taller tiene una razón específica de operar, no se trata únicamente de una clase impartida a un "grupo especial”, o para que "se ocupen" en algo, cada taller se planifica con cuatro objetivos: 1) estimular las funciones ejecutivas, para reducir el deterioro cognoscitivo, 2) trabajar las habilidades psicomotoras y de lenguaje, 3) promover el lazo comunitario a través de las aportaciones singulares, 4) construir puentes entre el huésped, su familia y lo social.

En este caso no se trata, ni se centra el objetivo en promover el arte desde su estricto marco académico -enseñanza del arte desde el discurso de la academia-, no se trata tampoco de que los huéspedes desarrollen habilidades artísticas, se trata, de integrar la peculiaridad de cada uno en un colectivo en donde lo que se produce es la creatividad integrativa, la sensibilidad y empatía hacia el otro semejante con quien se convive en un cotidiano.

Cada uno de los huéspedes de la comunidad aporta desde su propia invención y pequeñas construcciones creativas vías que establecen conexiones con el otro social. Sin embargo, ello no siempre resulta labor sencilla. Primero porque al trabajar de manera singular con cada caso es indispensable regular los intereses y pensamientos de suspicacia, así como que, no todos ellos tienen la misma edad, intereses o habilidades. Sin embargo, se establecen pautas objetivas y concretas en los proyectos comunitarios. Es función del equipo de psicólogos conjuntarlo e integrarlo. Segundo, porque al trabajar de manera comunitaria es indispensable planificar, diseñar y construir entre todos un proyecto el cual tenga una visión unificadora concreta en colectivo.

Algunos de los proyectos comunitarios que se han desarrollado <<entre varios $>>$ dentro de Casa de Medio Camino Querétaro- Casa Loohl, son: un falso documental (2018), exposiciones fotográficas (2019), programas de radio al interior de la comunidad (desde 2017), cosecha de plantas de huerto y producción de hogazas de pan para auto-consumo.

En el año 2020 durante el periodo de pandemia, se trabajó de manera simultánea con familiares -a distancia- y huéspedes de la comunidad en el libro Desquiciar la imaginación. Éste es el resultado 
de las invenciones que cada huésped construye como proyecto personal para hacer lazo social, tanto con su familia como con terceros. Se integraron varios factores desde la singularidad, lo colectivo, lo familiar e invitados externos; un esfuerzo, producto de la invención, intervención y aportación entre varios actores. En ese periodo también se concretaron tres libros individuales. Los cuales refieren intereses específicos de los huéspedes quienes participaron. Uno enfocado a la producción literaria de vivencias personales; otro en el que se re-escribe una autobiografía; y finalmente otro en el que se hace referencia a las crónicas de una etapa de vida. Estos tres libros han sido editados e impresos. Sin embargo, ello no podría haberse concluido sin el apoyo del equipo psicólogos, el esfuerzo de la familia por dar seguimiento al proyecto y el empuje, filosofía, seguimiento y soporte que proporciona el dispositivo para culminarlos.

\subsection{Imposibilidades clínicas y terapéuticas en la convivencia cotidiana}

Cada caso, plantea sus propias imposibilidades a nivel de estructura, sin embargo, ello implica un reto de escuchar y acompañar al huésped en sus tiempos, así como en sus habilidades. La figura semblante que adquiere cada uno de los psicólogos que se encuentra en la comunidad, es diferente, ello permite el trabajo desde distintas y múltiples transferencias. Cada psicólogo funge y activa diferentes formas de abordaje a los casos, pero de manera integral gracias a ello se pueden identificar los circuitos que se repiten, así como la orientación del caso. Las preguntas que de ahí se generaran respecto al caso clínico, dan lugar a la posibilidad de desdoblar en cierta medida, la certeza de la que en la estructura psicótica se adviene. Las estrategias en lo comunitario son vastas, sin embargo, la orientación que nos convoca es la línea sobre la que con intervenciones creativas y cotidianas se pretende recortar la invasión de lo real de lo que padece el psicótico (Rivas, 2006).

Otra de las imposibilidades que también se cifran en el trabajo en la comunidad terapéutica, es esencialmente con las familias. Más allá de un trabajo psicoeducativo, en la Casa de Medio Camino Querétaro, se trabaja con el duelo. El dolor subjetivo de las familias en tanto, la caída de las expectativas sobre ese sujeto que forma parte de ella. Nos queda claro que uno de los hilos que hacen el vínculo social, implica necesariamente a la familia. Por ello identificar, de qué manera y con qué recursos subjetivos ésta puede enlazarse a su familiar desde la propia estructura de la imposibilidad del caso. Los trazos que se construyen ahí, son los más delicados, debido a que, la familia, como sistema, tiene dinámicas, creencias, hábitos, costumbres y formas de relacionarse específicas, introducir variables que impliquen alterar ese sistema, resulta muchas de las veces un problema debido al ritmo y necesidades singulares. Sin embargo, cada caso presenta en su singularidad rasgos, con los que se trabajan porque provienen también de la familia.

El huésped puede mantener estabilidad y apego al tratamiento durante su estancia en la comunidad, sin embargo, si la familia y el cuidador primario no se involucran lo anterior no se logra sostener por si mismo en la mayoría de los casos. Es necesario el apoyo y acompañamiento con las familias y el huésped para que sostengan actividades, hábitos y conductas por lapsos de tiempo prolongado en un cotidiano. 
La familias en ocasiones presentan resistencias a este acompañamiento, las razones son claras: negación a cambiar rutinas y hábitos, negación al padecimiento de su familiar, ocupaciones diversas como trabajo, actividades personales. La culpa es un factor que se repite como parte del contexto sociocultural a la situación que se vive. En ocasiones es incomprensible desde la razón concebir que un familiar presenta un padecimiento psiquiátrico que lo imposibilita o detiene a dar continuidad a sus actividades. Ello se asocia culturalmente con "la locura", porque "debería salir adelante" o porque "no tiene una discapacidad física", "no le faltan brazos, piernas", "porque no tendría que estar mal si tiene todo lo necesario" estas son algunas de las ideas imaginarias -relacionadas con la completud física- que los familiares identifican como "razones" significativas, aunque producto de las construcciones culturales por las que en muchas de las veces se traducen en resistencias para asimilar, comprender y actuar frente a la enfermedad mental.

Sin embargo, se identifica con claridad que aquellas familias que se dejan acompañar y orientar son las que ya han atravesado por diferentes dispositivos, modelos, tratamientos, diagnósticos, que, desgastados económicamente, físicamente y emocionalmente, y habiendo aceptado la existencia del padecimiento y situación del familiar presentan mayor apertura al duelo entre las expectativas y la realidad que vive su familiar, ello permite una mayor apertura, así como estabilizaciones de mayor tiempo en los pacientes, así como los puentes posibles de comunicación y trato entre ellos. Sin embargo, lo anterior no es tarea sencilla. Es necesario trabajar en conjunto con la familia para identificar y diferenciar la situación, periodo y acontecimientos que han marcado puntos históricos de crisis, tratamientos e incluso de diferentes diagnósticos.

\section{Conclusiones}

Existen distintas formas de encierro, sin embargo, también existen dispositivos que brindan alternativas para los pacientes y sus familias, cada uno desde su acción permite que este concepto quede cada vez más alejado de las creencias y estereotipos en los que se ha cosificado a la enfermedad mental. La apertura a sensibilizarse desde otras perspectivas de acción y trabajo terapéutico que favorezcan a la singularidad de los lazos, posibilitan maneras de aliviar el malestar de la existencia y de buscar otras formas de abordar la enfermedad mental, más allá de generalizar la situación. El lazo social implica pensar cómo un sujeto puede vincularse, esto no es una acción automática, ni sistematizada, ni generalizada, es una forma de unirse con el otro semejante desde su singularidad y desde donde cada uno puede resolver de manera diferente.

Las comunidades terapéuticas son una vía para enlazar distintos saberes y trabajar rutas posibles para el tratamiento de la enfermedad mental, sin embargo, todavía existen brechas socioculturales que forman parte del imaginario colectivo y de prácticas que entrañan profundas creencias respecto la enfermedad mental que generan resistencias para pensar y reflexionar sobre las expresiones subjetivas del caso por caso y la habitabilidad del espacio cotidiano como una forma de trabajo que apunta a las relaciones de pluralidad, respeto y responsabilidad que tiene el paciente para lograr una mejor calidad de vida. 
El trabajo que se realiza dentro de la comunidad terapéutica Casa de Medio Camino Querétaro, es complejo principalmente por las resistencias fincadas por modelos asilares y asistenciales que han anclado como principio la concepción de la enfermedad mental como peligrosa, sin una solución o cura posible. Sin embargo, la principal herramienta con la que se trabaja en este dispositivo es con una praxis cualitativa, subjetiva y singular que en el día a día se emprende como parte de un proceso de concepción inseparable a la autonomía y funcionalidad posible que cada caso desde sus recursos subjetivos logra construir, haciendo uso de una red de apoyo. El soporte que aporta y brinda la comunidad terapéutica es el vínculo y el proceso de inclusión social, a partir de los esbozos de apropiación y abordamiento del problema "no" sin las complejas características de interacción que permite el trabajo en grupo y en colectivo. Teniendo presente la alteridad y la responsabilidad ética de cada los miembros que la constituyen.

Aunque a nivel internacional y organismos como la Organización Panamericana de la Salud OPS recomiendan las comunidades terapéuticas como modelo de trabajo, así como plantea que "la atención en la comunidad logra una mayor efectividad terapéutica además de ser humanizante" (OPS, 2017) también plantea que "por otro lado los usuarios de los servicios de salud mental prefieren vivir fuera de las instituciones gozando de sus derechos como ciudadanos" la desinstitucionalización de la salud mental implica pensar nuevas prácticas y servicios alternativos los cuales han demostrado ser más eficaces, efectivos y humanos. Sin embargo, para el seguimiento y procesos de inclusión social es necesario de una red de apoyo que permita tanto a las familias, al paciente y al cuidador primario un punto de base para dar seguimiento, autonomía y funcionalidad. Hacerlo en solitario, conlleva a formas de encierro involuntarias que en ocasiones por desinformación o temor generan prácticas complejas dentro del sistema familiar.

Cada dispositivo desde sus bases, su función y estructura puede brindar orientación, apoyo y seguimiento, sin embargo, la complejidad de sostener un trabajo interdisciplinario es en muchas de la ocasiones harto difícil debido a los factores que intervienen de manera contingente, ambiental e indirecta.

El trabajo al interior de una comunidad terapéutica, implica el enorme esfuerzo de diversos actores los cuales tienen un objetivo en común, cuando éste se pierde o se centraliza desde la atención masiva el planteamiento nuclear de la comunidad terapéutica tiende a tomar un rumbo asilar o asistencial. Una de las vías de trabajo principal de una comunidad terapéutica, depende en gran medida de la visión, orientación y composición de su estructura pensada para brindar herramientas para la autonomía y funcionalidad del paciente.

De lo que se ha pretendido con esta brevísima exposición, es de dar testimonio de las experiencias y procesos que implica la convivencia en lo cotidiano en la comunidad terapéutica, un modelo que si bien parte de las construcciones sociales que históricamente han derivado desde la segunda mitad del siglo XX, la Casa de Medio Camino Querétaro, da cuenta de su labor y de lo que ahí se produce tanto clínica como comunitariamente. 


\section{Referencias}

Alcuaz, C. (2021). Otra sociedad para la locura: Estudio sobre los lazos sociales en la psicosis. Xoroi.

Aragón, L. (2011). El Testimonios y sus aporías. Escritura e imagen. Extra, 295-311, https://doi.org/10.5209/ rev_ESIM.2011.37740

Basaglia, F. (1972) La institución negada. Informe de un hospital psiquiátrico. Ediciones Corregidor.

Buttler, J. (2010). Marcos de guerra. Paidós.

Comas, Domingo. (2007). Comunidades Terapéuticas en España. Situación actualy propuesta funcional. Grupo GID.

Capponi, S. (2011). Para una genealogía de la psiquiatría ampliada. Cuadernos Brasileños de Salud Mental. 3 (6),106-125, https://periodicos.ufsc.br/index.php/cbsm/issue/view/2975

Crespo, M \& Campo, Maribel. (2003). Historia de la clasificación internacional del funcionamiento de la discapacidad y de la salud (cif): un largo camino recorrido. Revista española sobre discapacidad intelectual, 34(205), 20-26.

De Certeau, M. (2007). El lugar del otro. Katz.

De Certeau, M. (2006). La escritura de la historia. UIA.

Derridá, J. (1972). Márgenes de la filosofía. Cátedra.

OPS (2020). Desinstitucionalización de la atención psiquiátrica en América Latina y el Caribe. Organización Panamericana de la Salud. https://cutt.ly/7YhUIQi

Foucault, M. (1991). Saber y verdad. La piqueta.

Foucault, M. (1978). La verdad y las formas jurídicas. Gedisa.

Frenk, J. (2008). Para entender el sistema de salud de México. Nostra.

Heidegger, M. (1997). Arte y poesía. Fondo de Cultura Económica.

Huertas, R. (2011). En torno a la construcción social de la locura. lan Hacking y la historia cultural de la psiquiatría. Revista de la Asociación Española de Neuropsiquiatría, 31(11), 437-456, https://dx.doi. org/10.4321/S0211-57352011000300004

Secretaría de Salud de México, Organización Panamericana de la Salud, Organización Mundial de la Salud (2011). Informe de evaluación del sistema de salud en México utilizando el Instrumento de Evaluación para Sistemas de Salud Mental de la Organización Mundial de la Salud Secretaría de Salud de México, Organización Panamericana de la Salud, Organización Mundial de la Salud https://cutt.ly/dYhU1jC

Jones, M. (1956). The Concept of a Therapeutic Community. The American Journal of Psychiatry. 8(112), 647-650, https://doi.org/10.1176/ajp.112.8.647.

Laning, R., \& Cooper, D. (1978). Razón y violencia. Paidós.

Marcos, S., \& Gomezjara, F. (1982). Dossier México: sobre alternativas a la psiquiatría. Nueva sociología.

Norma Oficial de la Secretaría de Salud Mexicana NOMo25-SSA2-2014, Para la prestación de servicios de salud en unidades de atención integral hospitalaria médico-psiquiátrica, de 04 de septiembre de 2015, pp, 4 a 14.

Novella, E. (2008). Del asilo a la comunidad: interpretaciones teóricas y modelos explicativos. Frenia, 8(1), 9-32, http://www.revistaaen.es/index.php/frenia/article/view/16454

Pérez del Río, F. (2010). Origen alemán de la comunidad terapéutica. Revista de la Asociación Española de Neuropsiquatría, 30(105), 145-149 
Porter, R. (2003). Breve historia de la locura. Fondo de Cultura Económica.

Ríos, A. (2016). Como prevenir la locura. Siglo XXI.

Rivas, E. (2006). Pensar la psicosis. El trato con la disidencia psicótica o el diálogo con el psicótico disidente. Gramma.

Tejeda Ruíz, C. (1967). Los hospitales granjas. Salud Pública de México, 9(4), 587-59o.

Sacristán, C \& Ordorika, T. (2018). Historia de la Psiquiatría en México. Voces, testimonios e imágenes de sus protagonistas. Láser.

Sutton, S. (2006). La exclusión social y el silencio discursivo. Iberóforum, 1(2), 1-10

Stucchi, S. (2013). Estigma, discriminación y concepto de enfermedad mental. Revista de Neuro- Psiquiatría, 76(4), 218-223, https://doi.org/10.20453/rnp.v76i4.1170

\section{AUTORES}

Sandra G. Rosas Landa Zamudio. Doctoranda en Humanidades de la Salud por la UNAM, Facultad de Medicina. Maestra en Arte Contemporáneo, Maestría en Saberes sobre Subjetividad y Violencia. Docente de cátedra en la Universidad Autónoma de Querétaro en la Facultad de Arquitectura.

Israel B. Aguillón Cruz. Licenciado en Psicología de la Universidad Autónoma de Querétaro. Acompañante Terapéutico independiente. Promotor y coordinador de espacios para el trabajo protegido de pacientes psiquiátricos en Querétaro. 